\title{
A posterior semi-stochastic low Earth debris on-orbit breakup simulation model
}

\author{
A.K. Anilkumar ${ }^{\mathrm{a}, *}$, M.R. Ananthasayanam ${ }^{\mathrm{b}}$, P.V. Subba Rao ${ }^{\mathrm{a}}$ \\ a Applied Mathematics Division, Vikram Sarabhai Space Centre, Trivandrum 695022, India \\ ${ }^{\mathrm{b}}$ Department of Aerospace Engineering, Indian Institute of Science, Bangalore 560012, India
}

\begin{abstract}
A posterior modeling approach for on-orbit breakup, named ASSEMBLE (A Semi-Stochastic Environment Modeling of Breakup in LEO), is proposed. The main objectives of this paper are the statistical characterization of the orbital and physical parameters of the fragments generated by an on-orbit breakup. The analysis of two line element (TLE) data of the fragments of some fragmentation events, such as the Indian PSLV-TES rocket body breakup, showed their orbital parameters obey statistical distributions. The apogee or perigee height, limited by breakup altitude, and the inclination follow Laplace distributions, while the eccentricity a Lognormal distribution. Subsequently, suitable empirical relations in the literature help to expand the fragmentation scenario to mass, effective area, and ballistic coefficient. Lastly, the simulation of well-known historical breakups of (i) SPOT I rocket body, (ii) COSMOS 1813, (iii) STEP II rocket body, and (iv) CBERS I/ SAC I provide further confidence in the present model for orbital debris studies.
\end{abstract}

\section{Introduction}

Since the first satellite breakup in 1961 to date, more than 175 spacecraft and rocket bodies have fragmented in orbits $[1,2]$. The resulting debris fragments constitute nearly $40 \%$ of the 9000 or more catalogued objects. The catalogued fragment count does not,

\footnotetext{
* Corresponding author. Tel.: +91471 2565629; fax: +914712704134 .

E-mail addresses: anilk_ak@yahoo.com (A.K. Anilkumar), sayanam@aero.iisc.ernet.in (M.R. Ananthasayanam), pulipakasrao@yahoo.co.in (P.V. Subba Rao).
}

however, include the numerous fragments that are too small for tracking. In order to describe the orbital debris environment, it is necessary to develop mathematical models to simulate the fragments resulting from explosions/collisions. Such models facilitate a realistic assessment of the risk due to the debris clouds. A posterior model, ASSEMBLE (A Semi-Stochastic Envioronment Modeling of Breakup in LEO), based on the available information on the breakup event and characterization of orbital parameters is proposed. This model utilizes probability distributions for orbital characteristics of the fragments and empirical relations for the physical characteristics and assigns the 


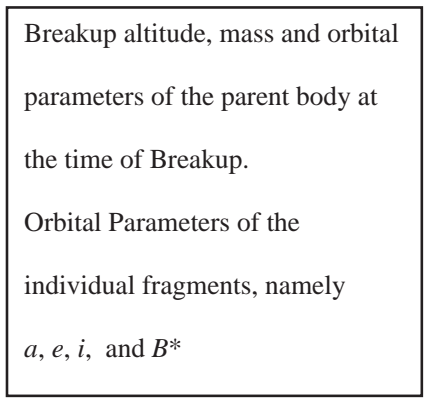

Aspect block - 1

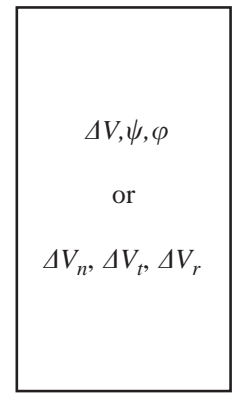

Aspect block - 2
Empirical relations

Connecting

Number, Mass ( $m)$, Size,

Effective Area $(A)$ and

Ballistic Coefficient $(B)$

Aspect block - 3

Fig. 1. Three aspects of orbital breakup modeling and analysis.

fragment masses and sizes through the magnitude of incremental velocity $\Delta V .^{1}$ Subsequently it has been applied to simulate some typical historical on-orbit fragmentation events. In the present approach the distribution of fragment incremental velocities is a consequence rather than imposed through empirical relations as is done in the earlier approaches.

\subsection{Conceptual basis for the present model}

Any of the modeling processes, considered as a physical theory, obviously have three approaches, namely, intuitive beginning, mathematical modeling, and consistency with experiments. These are similar to the various approaches in probability theory [3], and have increasing amount of information. The mathematical modeling of the breakups of objects in space is quite a difficult task due to the randomly varying ways in which the combination of the orbital parameters (through the debris velocity), mass, size, and shape can occur. It is better to depend far more on the debris fragment measurements in the mathematical modeling of such processes.

This paper deals with a modeling approach to the on-orbit breakup of an object in space and is based on an analysis of the available catalogued data, as is generally provided by US Space Surveillance Network (US SSN), of the fragments soon after the breakup. On-orbit breakups create many fragments and each has its orbital characteristics such as the semi-major axis, eccentricity, inclination, and other parameters such as

\footnotetext{
${ }^{1}$ See Nomenclature in Appendix A at the end of the paper.
}

size, shape, the ballistic coefficient, and so on. The catalogued data provide only the orbital characteristics of fragments besides a $B^{*}$ term related to ballistic coefficient $B$ with $B^{*}=\left(\rho_{0} / 2\right) B$. The size and shape cannot be easily estimated on the basis of these characteristics and hence need to be obtained from empirical relations based on some laboratory experiments. These relations provide estimates based on samples only and not those of the population. But all these variables characterizing the fragments are in some way connected due to the underlying physical process in an explosion and one cannot assign arbitrary random values for their various properties. The question is from which of the properties can one commence with and later derive the others? This is similar to obtaining the height, weight, and age of a person. Where do we start for best possible estimates? It turns out that there is a hierarchy among the variables of a system. The hierarchy, in terms of whatever it implies, dictates in the present case to commence from the independent variables such as the semi-major axis and eccentricity, to arrive at the velocity change rather than proceeding the other way round as has been generally done in the earlier approaches.

\subsection{Earlier approaches of breakup modeling}

Fig. 1 shows the three aspects of any modeling effort for the orbital debris breakup. Block 1 corresponds to the orbital characteristics of the fragments, while Block 2 includes the distribution of the velocity of the fragments and is the focus of the entire breakup modeling by different approaches by the way this aspect 
is handled. Block 3 relates the number of fragments, their individual mass, and size through empirical relations obtained from laboratory/terrestrial experiments.

In the earlier approaches [4,5] one commences from Block 2 and proceeds to Block 1 to simulate the orbital characteristics. Block 3 characteristics are independently simulated and are related to Block 2 through empirical relations connecting size or area to mass ratio $A / m$ to $\Delta V$, the incremental velocity.

The magnitude of the incremental velocities of the fragments is assumed to obey certain distributions such as a Gaussian distribution. Further, their directionality in space is assumed as uniformly distributed in azimuth $\psi$ and elevation $\varphi$. In the absence of any information, in the first instance, this would be natural and rightly so. However, there is the difficulty of the choice of the parameters of distribution. In general, the fragment velocities would obviously depend on the breakup location (true anomaly $\theta$ ), intensity and also the directionality of the explosion. In some other approaches [6,7], simulation commences from Block 3 , namely the relations firstly connecting the number of debris and mass and secondly the mass to the size, and then proceeds to derive Block 2 properties. It is based on the size (or area to mass ratio) versus velocity relationships. Further, since these relations cannot be deterministic it uses the well-known triangular distribution [8] to impart additional random velocities over the above. Later, Block 1 simulation is carried out utilizing the information from Block 2. Since it is well-known that the evolution of the debris with time depends on the orbital parameters and the ballistic coefficient, one can expect uncertainties or inaccuracies in these models due to the use of many intermediate empirical relations in these procedures $[4,6]$ as well. The recent NASA's breakup model $[9,10]$ incorporated in MASTER 2001 differing from MASTER ' 99 considers a bimodal normal distribution to derive area to mass from diameter and a Lognormal distribution depending on $A / m$ is applied to spread the incremental velocities of the fragments. It was noticed that as a consequence the clouds remain centered much more around the parent body orbit. It may also be noted that the above approaches do not appear to utilize the available orbital information of the breakup such as the Gabbard diagram together with the recorded deviations in the periods and inclination or the catalogued TLE data. The present approach being based on the available information on breakup, the observations on Lognormal nature of incremental velocities and clustering of fragments around the parent body follow as consequences.

\section{Breakup of the Indian PSLV-TES mission spent upper stage}

The basic structure of the present model has been based on an analysis [11] of the characteristics of the parameters of fragments of the breakup in low Earth orbit (LEO). As an illustration, the recent on-orbit breakup of the spent upper stage of the Indian PSLVTES Mission, launched on 22 October 2001, from Sriharikota Range of the Indian Space Research Organization, is considered. After the successful completion of the launch vehicle mission, the spent upper stage of PSLV was inserted into an orbit of nearly $675 \mathrm{~km} \times$ $550 \mathrm{~km}$ at an inclination of $97.9^{\circ}$. This rocket body (with international designation: 2001049D and mass about $885 \mathrm{~kg}$ ) exploded on 19 December 2001 at about $1140 \mathrm{~h} \mathrm{UTC}$. The resulting fragments were tracked and catalogued as TLE elements by USSPACECOM. The details of this breakup event [2] are

$$
\begin{array}{ll}
\text { Breakup location: } & \sim 25^{\circ} \mathrm{S}, 340^{\circ} \mathrm{E} \\
\text { Altitude: } & \sim 670 \mathrm{~km} \\
\text { Pre-event orbit: } & \sim 550 \mathrm{~km} \times 675 \mathrm{~km}, 97.9^{\circ} \\
& (\text { inclination }) \\
& (a=6990.5 \mathrm{~km}, e=0.0089)
\end{array}
$$

Number of

catalogued objects: $>300$

Fig. 2 provides the Gabbard diagram of the 301 fragments after nearly 30 days of breakup. The slight coalescence in the left arm of the Gabbard diagram is due to the decay of the orbits of the fragments in 30 days. Analysis of the orbital characteristics summarized in Fig. 3 shows the Laplace distribution [12] fit for apogee height, Lognormal fits for eccentricity and the ballistic parameter $B^{*}$ values and Laplace fit for the inclination as appropriate. Analysis of goodness of fit tests [13] carried out for all the above cases in terms of $\chi^{2}$ values, $\chi^{2}$ being a statistical measure of the agreement between the observed frequencies and the expected frequencies and Kolmogorov-Smirnov statistics, clearly brought out the adequacy of the respective fits. 


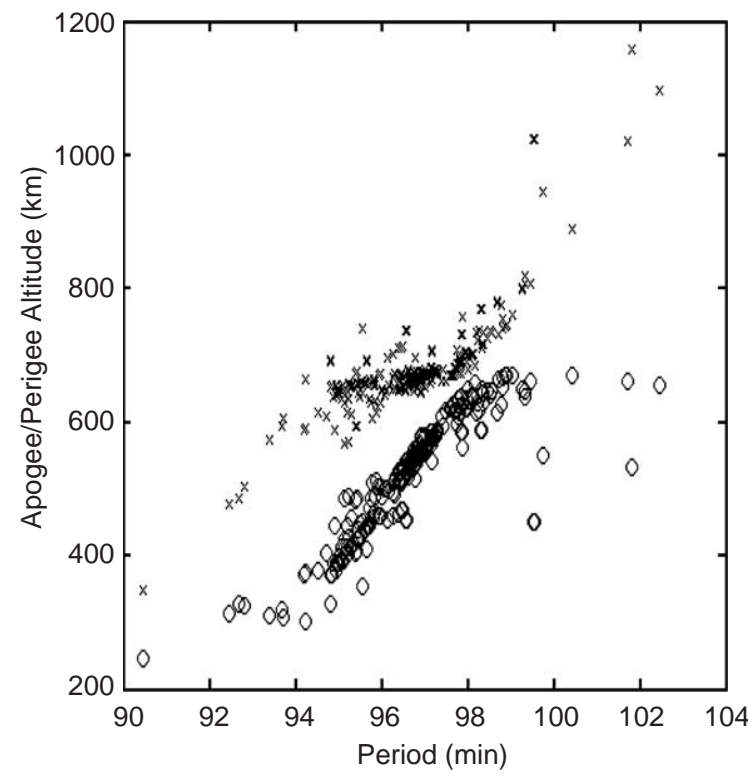

Fig. 2. The Gabbard diagram from the TLEs of PSLV-TES spent upper stage breakup fragments after 31 days of breakup.

\section{The approach in the ASSEMBLE model}

Accordingly, the present model commences with the characterization of the apogee/perigee heights (restricted by the breakup altitude), inclination and eccentricity in terms of, respectively, Laplace and Lognormal distributions. The location parameters of these distributions correspond to the breakup point and their scale parameters depend on the intensity of explosion [11]. The distribution of the $B^{*}$ parameter of the catalogued objects was also found to follow a Lognormal distribution. Thus, all the necessary information in Block 1 is available and one can proceed from here to derive the detail of Block 2. Information of Block 3 needs to be simulated on the basis of the state-ofthe-art empirical relations or their updates $[9,10]$. For example, the relations that can be used in this context are given in Appendix B. Proper assignment of the orbital characteristics to the physical characteristics utilizing the information in Block 2 can then be carried out. Thus, this model can be seen to be semi-stochastic in nature capable of simulating all the required details of the fragments. Hence, the proposed procedure utilizes probabilistic distributions for orbital characteristics of the fragments and empirical relations for the physical characteristics and relates them through the magnitude of $\Delta V$. Further, as the characterizations are based mainly on information in LEO breakups, this model is named ASSEMBLE.

For the purpose of simulating earlier breakups, generally one has the information of the Gabbard diagram and the information on the number of tracked fragments, maximums of deviation in the periods $\Delta P$ and deviation in the inclinations $\Delta i$, and other details about the parent body at the time or just prior to the breakup event [1], but not all the quantitative orbital and physical characteristics of the fragments. It is demonstrated that ASSEMBLE is able to simulate the debris clouds in a statistical way by generating the fragmentation events by suitable choice of the parameters involved in this procedure. It is shown further that the characterization of the distribution of the ballistic coefficients helps to provide an improved description of the evolution of the debris clouds with time.

Fig. 4 provides a detailed flow chart of the ASSEMBLE model. It may be pointed out that if the velocities of the fragments are assumed to be normal, or derived otherwise from the size distributions as in other approaches, it turns out that, in particular, the eccentricity and the $\Delta V$ do not follow the Lognormal distributions and hence are inconsistent with the respective information of the catalogued data.

In order to simulate a typical breakup using the present model, the information about the breakup altitude or breakup true anomaly, apogee and perigee height (equivalently $a$ and $e$ ), inclination at the time of breakup and also mass of the parent body are necessary. Apart from these one needs the range of apogee or perigee heights and maximum $\Delta P$ and maximum $\Delta i$. For simulating the historical breakups, this information is readily available in the literature [1]. These are very crucial and provide the scale parameters for the statistical distributions.

It is well known that the apogee and the eccentricity are related to radial and transverse components of the velocity, while the inclination and the right ascension of the ascending node are related to the normal component. Appendix $\mathrm{C}$ provides the selection of scale parameters based on the probability distributions and the maximum change observed in these parameters. In view of the uncertainties in the input there is a necessity for some subjective tuning of the scale parameters. It is assumed here that the observed spread 

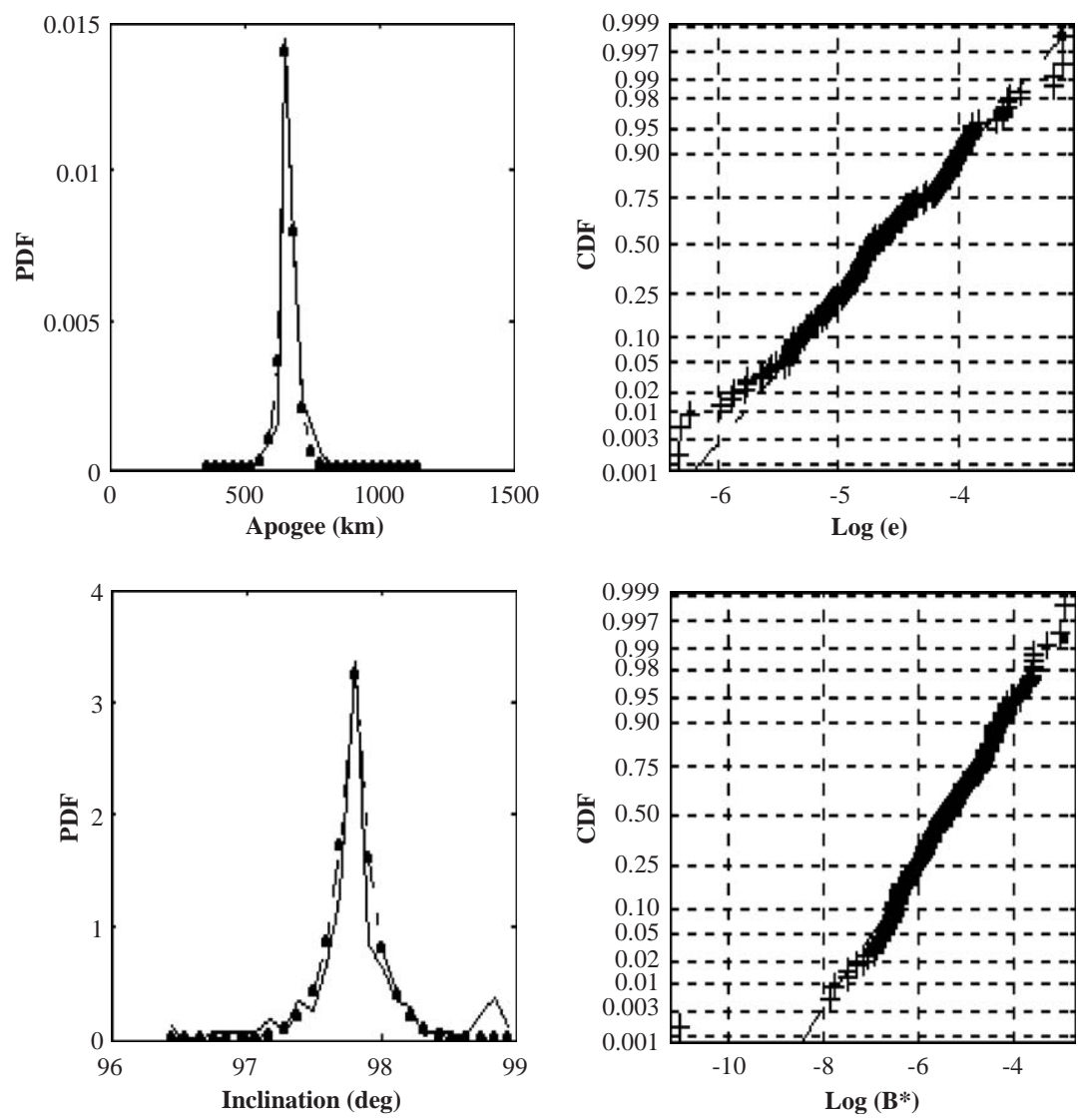

Fig. 3. The distribution of parameters from the TLEs of PSLV-TES spent upper stage breakup fragments after 31 days of breakup (*, + data, - fit) PDF- probability density function CDF-cumulative distribution function.

in the Gabbard diagram corresponds to a $99 \%$ confidence value of the random variable.

\subsection{Steps for simulating a typical breakup}

The basic procedure of ASSEMBLE can be summarized in the following steps.

1. The location parameters for apogee/perigee altitudes and inclination distributions, both of which follow generally a Laplace distribution, and the eccentricity which obeys a Lognormal distribution can be obtained from the breakup point details of the parent body. Next, based on the ranges of apogee/perigee heights and available maximum $\Delta P$ and $\Delta i$, one can obtain scale parameters (Appendix $\mathrm{C}$ ). The number of fragments generated, namely $N$, and the mass of individual fragments can be obtained by using the number versus mass relationship $[6,14]$ such as the one provided in Appendix B or any other appropriate updates of relations for an explosion $[9,10]$.

2. Generate $N$ random sets of the apogee or perigee height, inclination and eccentricity combinations from the respective Laplace and Lognormal distributions, by restricting the apogee height or perigee height bounded by breakup altitude.

3. Using the above sampled values of the apogee/ perigee heights and eccentricity calculate the true anomalies of each of the fragments at the breakup point.

4. Obtain the velocity imparted to each of the fragments and assign the additional delta velocities to the masses (or area to mass ratio), based on triangular distribution constraint or any other method that is found suitable. 


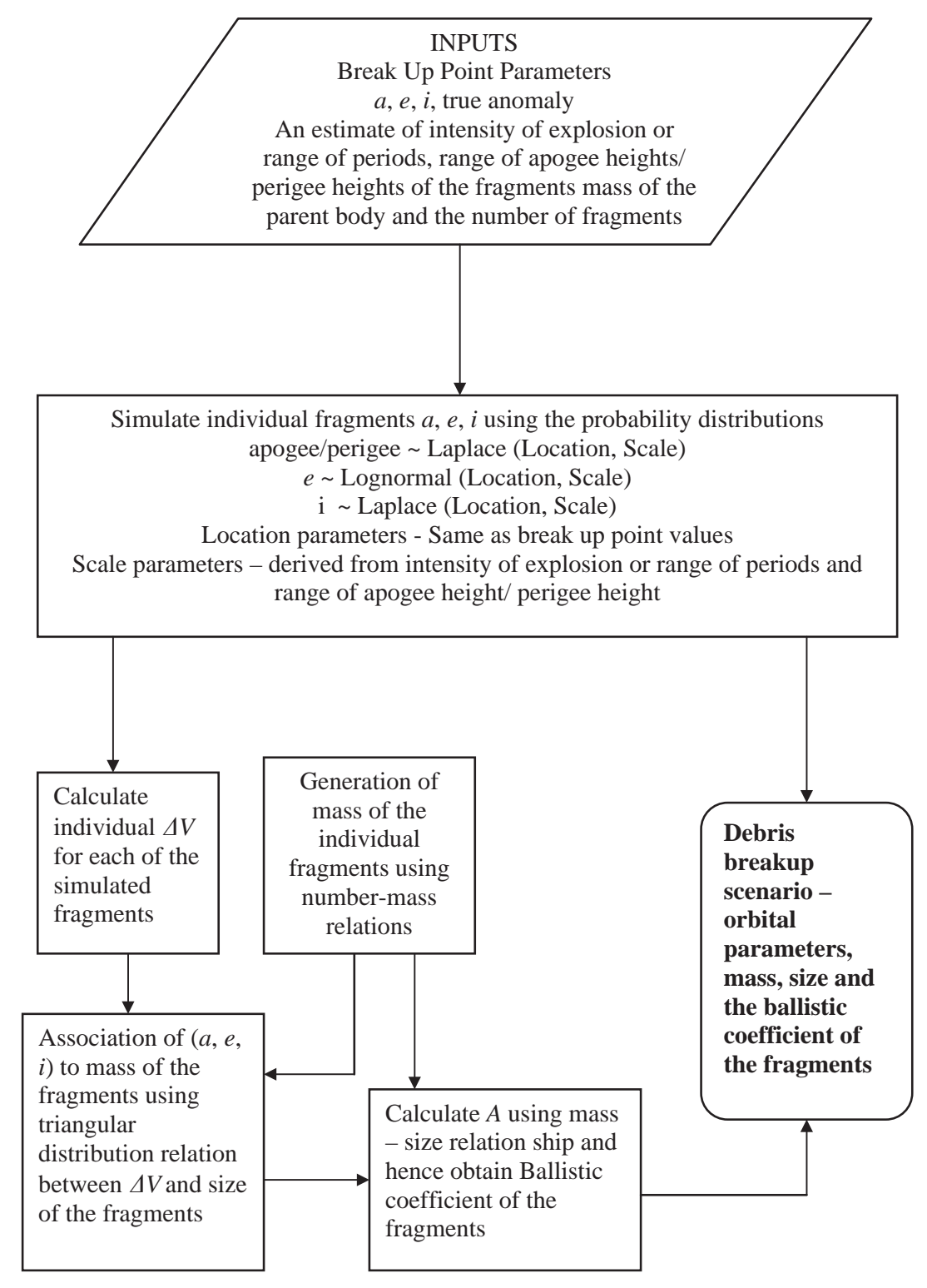

Fig. 4. Flow hart of the ASSEMBLE model to simulate a breakup.

It is pointed out that these orbital parameters in Step 2 follow a joint distribution with marginal distributions as described. Hence, while simulating these, the cross-coupling between the parameters can be taken into account. But the multiple correlations between these parameters are mostly unknown. However, the assumption of zero correlation if assumed, and each of the parameters independently simulated from the marginal distributions and randomly coupled, does not significantly alter the fit characteristics as an ensemble.

\subsection{Simulation of some historical breakups using ASSEMBLE model}

In this section, we describe the results based on the simulation of some of the typical historical on-orbit 
Table 1

Details of the analyzed breakup events

\begin{tabular}{|c|c|c|c|c|}
\hline Title & STEP II rocket body & CBERS-1/SACI-1 rocket body & COSMOS 1813 & SPOT I rocket body \\
\hline International designation & 1994-029B & 1999-57C & 1987-004A & 2001-049D \\
\hline Catalogue number & 23106 & 25942 & 17297 & 26960 \\
\hline \multicolumn{5}{|l|}{ Satellite data } \\
\hline Type & Pegasus HAPS & Long March 4 3rd stage & Payload & Ariane 1 3rd stage \\
\hline Owner & USA & PRC & CIS & ESA \\
\hline Launch date & 19 May 1994 & 14 October 1999 & 15 January 1987 & 22 February 1986 \\
\hline Dry mass $(\mathrm{kg})$ & 97 & 1000 & 6300 & 1400 \\
\hline \multicolumn{5}{|l|}{ Event data } \\
\hline Date & 3 June 1996 & 11 March 2000 & 29 January 1987 & 13 November 1986 \\
\hline Location (deg) & $67 \mathrm{~S}, 56 \mathrm{E}$ & $51.2 \mathrm{~S}, 48.5 \mathrm{~W}$ & $73 \mathrm{~N}, 122 \mathrm{E}$ & $7 \mathrm{~N}, 42 \mathrm{E}$ \\
\hline Time & 1518 GMT & 1304 UTC & 0555 GMT & 1940 GMT \\
\hline Altitude (km) & 625 & 741 & 390 & 805 \\
\hline \multicolumn{5}{|l|}{ Pre-event elements } \\
\hline Mean anomaly (deg) & 108.3711 & 43.0989 & 178.1696 & 300.1947 \\
\hline Right ascension (deg) & 197.8565 & 145.5131 & 256.7724 & 18.0087 \\
\hline Mean motion (rev/day) & 14.56780581 & 14.46866365 & 15.60427 & 14.2216 \\
\hline Inclination (deg) & 81.9749 & 98.5373 & 72.8163 & 98.6973 \\
\hline Eccentricity & 0.0165742 & 0.0012467 & 0.0043147 & 0.0021203 \\
\hline Arg. of Perigee (deg) & 249.9583 & 316.9224 & 182.01 & 60.1312 \\
\hline \multicolumn{5}{|l|}{ Debris cloud data } \\
\hline Maximum $\Delta P(\min )$ & 27.9 & 10.985 & 9.1 & 6.2 \\
\hline Maximum $\Delta i(\mathrm{deg})$ & 2.4 & 0.99 & 0.1 & 1.2 \\
\hline No. of catalogued fragments & $\sim 713$ & $\sim 300$ & $\sim 846$ & $\sim 488$ \\
\hline
\end{tabular}

Table 2

Location and scale parameters for various breakups

\begin{tabular}{|c|c|c|c|c|c|}
\hline \multirow{2}{*}{$\begin{array}{l}\text { Orbital parameters } \\
\text { at breakup }\end{array}$} & \multirow{2}{*}{$\begin{array}{l}\text { Parameters of } \\
\text { the distribution }\end{array}$} & \multicolumn{4}{|c|}{ Fragmentation event } \\
\hline & & $\begin{array}{l}\text { STEP II } \\
\text { rocket body }\end{array}$ & $\begin{array}{l}\text { CBERS-1/SACI-1 } \\
\text { rocket body }\end{array}$ & $\begin{array}{l}\text { COSMOS } \\
1813\end{array}$ & $\begin{array}{l}\text { SPOT I } \\
\text { rocket body }\end{array}$ \\
\hline Perigee height $\sim$ & Location & 500 & 726 & 360 & 805 \\
\hline $\mathrm{LP}^{\mathrm{a}}(\mathrm{km})$ & Scale & 85 & 100 & 100 & 100 \\
\hline Eccentricity $\sim$ & Location & -4.1 & -6.6 & -5.5 & -6.2 \\
\hline $\mathrm{LN}^{\mathrm{b}}$ & Scale & 0.67 & 1.35 & 1.1 & 1.0 \\
\hline Inclination $\sim$ & Location & 82 & 98.5 & 72.82 & 98.7 \\
\hline $\mathrm{LP}^{\mathrm{a}}(\mathrm{deg})$ & Scale & 0.6 & 0.55 & 0.03 & 0.4 \\
\hline
\end{tabular}

${ }^{\mathrm{a}} \mathrm{LP}$-Laplace distribution.

${ }^{b} \mathrm{LN}$-Lognormal distribution.

fragmentation events. Table 1 provides the important details of these events and Table 2 provides the details of the parameters utilized in the ASSEMBLE model with their location and scale parameter values for simulating and analyzing the four fragmentation events. 


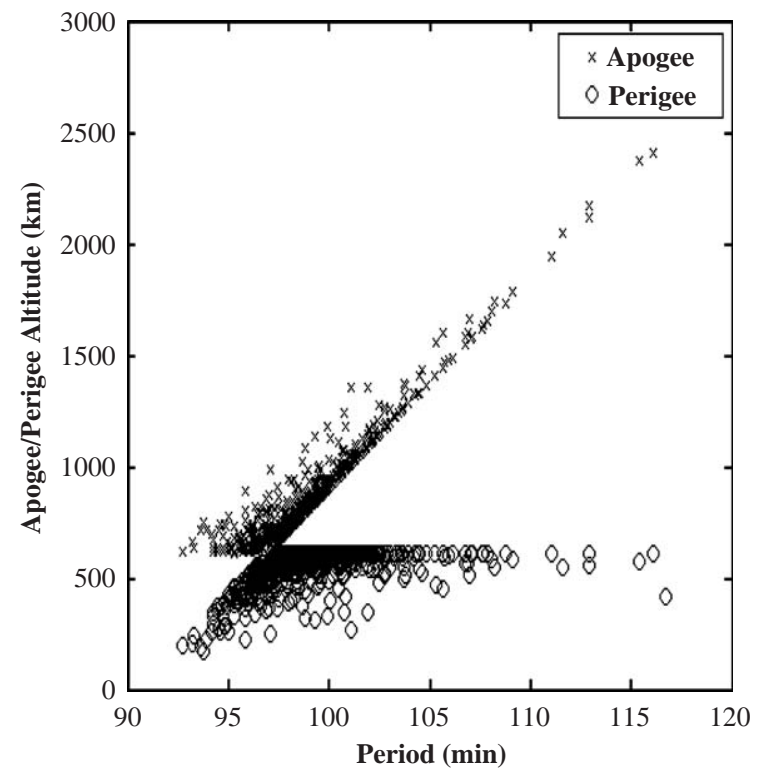

Fig. 5. Step II rocket body debris clouds of 713 fragments at the time of breakup simulated using ASSEMBLE model.

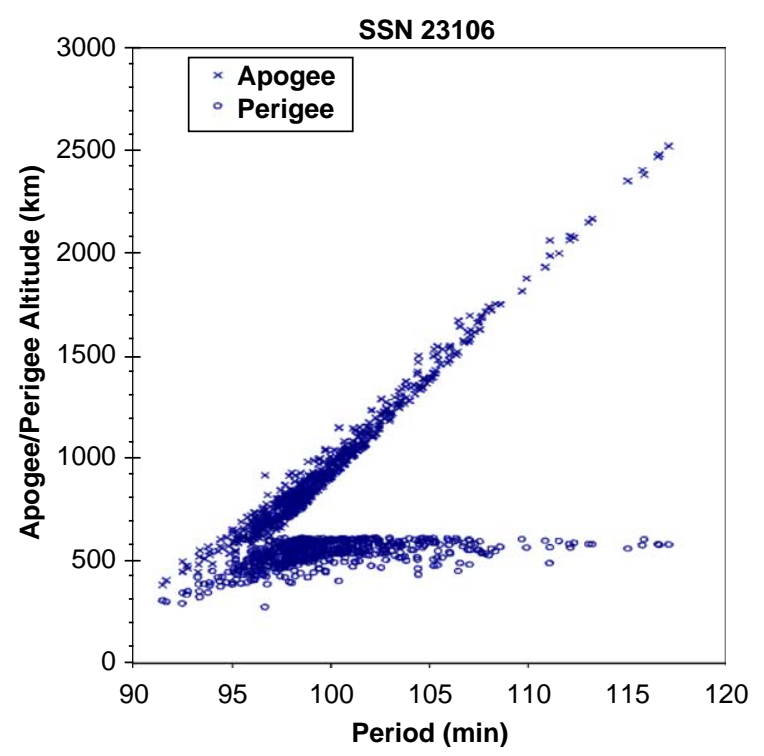

Fig. 6. Step II rocket body debris clouds of 713 fragments as of August 29, 1996 (89 days after the breakup) as reconstructed from US SSN database $[1,15]$.

\subsubsection{Case study of STEP II rocket body}

Fig. 5 provides the Gabbard diagram of the debris clouds at the instant of breakup simulated by ASSEMBLE model, while Fig. 6 provides the Gabbard dia-

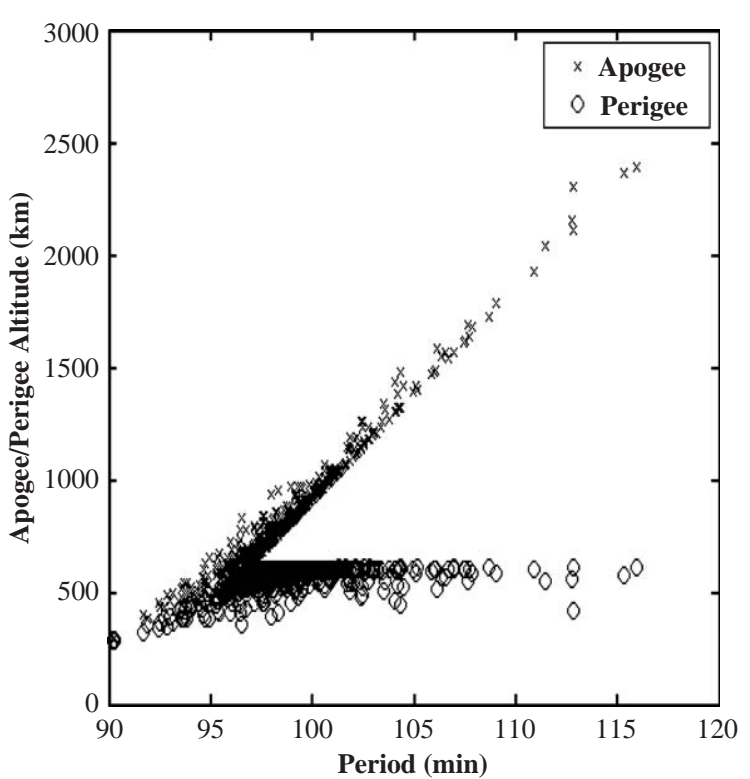

Fig. 7. Step II rocket body debris clouds of 713 fragments after 89 days of breakup simulated using ASSEMBLE model.

gram of the 713 fragments as of August 29, nearly 90 days after the breakup [1,15]. The differences in the left arms of the apogee height between Figs. 5 and 6 can be attributed to the decay effect in 90 days. In order to demonstrate this fact, the simulated fragments were propagated for 90 days and the resulting Gabbard diagram is shown in Fig. 7. Now it can be seen that Figs. 6 and 7 are matching well. The propagation routine used is the one which considers only the drag effect of the atmosphere [16]. Figs. 8-12 provide extensively the various other characteristics of the fragments as simulated by the ASSEMBLE model. It is noted that the features shown in these figures are matching well with those provided by the analysis of Anz-Meador [17] about the STEP II rocket body (1994-029B) breakup. As expected, it can be seen that the derived quantities like $\Delta V$ and the ballistic coefficient show their nature as Lognormal distribution.

\subsubsection{Case study of CBERS-I/ SACI-1 rocket body}

Fig. 13 provides the Gabbard diagram of the above breakup $[1,8]$ as reconstructed from US SSN database just after a day of the breakup. The Gabbard diagram of the fragments simulated using the present model just at the time of breakup is provided in Fig. 14. Close match of these two figures canan be easily noticed. 

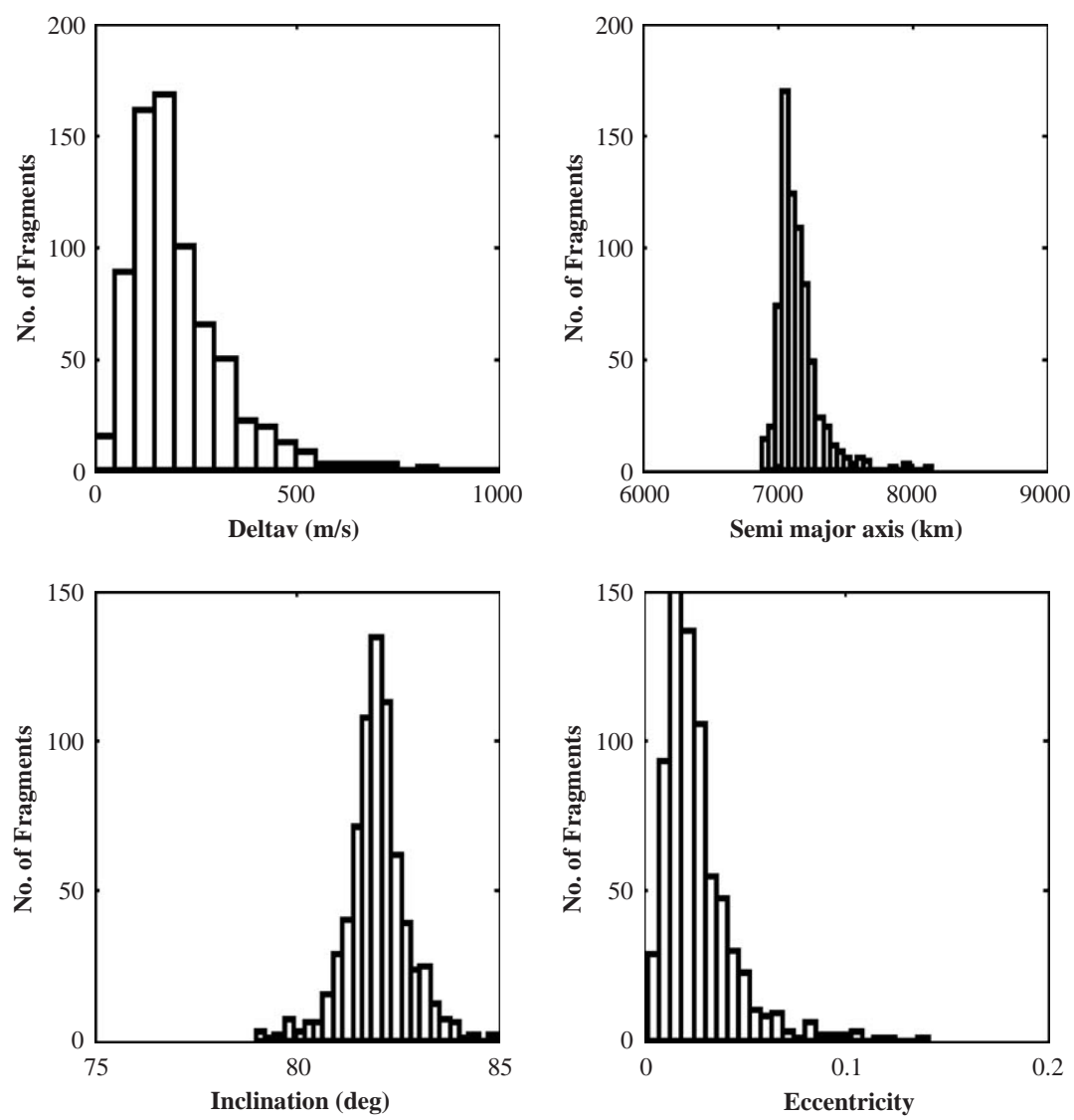

Fig. 8. Characteristics of the simulated breakup fragments of Step II rocket body.

\subsubsection{Case study of COSMOS 1813}

Fig. 15 provides the Gabbard diagram of Cosmos 1813 debris cloud for 846 fragments $[1,19,20]$ as reconstructed from PARCS radar observations taken about $10 \mathrm{~h}$ after the breakup. Fig. 16 shows the simulated Gabbard diagram after $10 \mathrm{~h}$ of evolution of the debris clouds using ASSEMBLE model. As the breakup occurred at a very low altitude, the evolution of debris clouds for $10 \mathrm{~h}$ brings a change in the left arms of the Gabbard diagram. Fig. 17 provides a Gabbard diagram of the fragments $10 \mathrm{~h}$ after the event as simulated using the model proposed by Jehn [6] and is extracted from Ref. [6], whose left arm does not show much change since breakup. The comparison between Figs. 15, 16 and 17 clearly brings out the ability of the ASSEMBLE model better than the other approaches in simulating the debris clouds.

\subsubsection{Case study of SPOT I rocket body}

Fig. 18 provides the Gabbard diagram of the debris cloud of SPOT I rocket body after 3 months of evolution as reconstructed from US SSN database [1,21] and Fig. 19 provides the Gabbard diagram corresponding to the fragments after 3 months of propagation simulated by the ASSEMBLE model. It is clear that ASSEMBLE simulates the debris clouds to obtain a good match with the observations.

\section{Conclusions}

A posterior model, ASSEMBLE, based on the available information on the breakup event and characterization of orbital parameters is proposed. This procedure utilizes probabilistic distributions for orbital characteristics of the fragments and empirical relations 


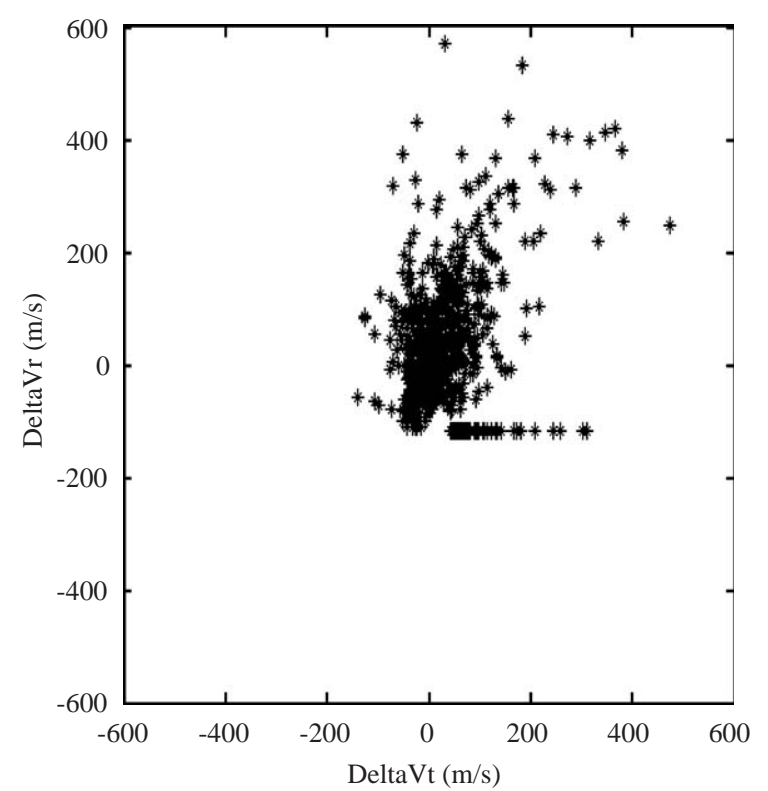

Fig. 9. $\Delta V t$ vs. $\Delta V r$ of the simulated breakup fragments of Step II rocket body.

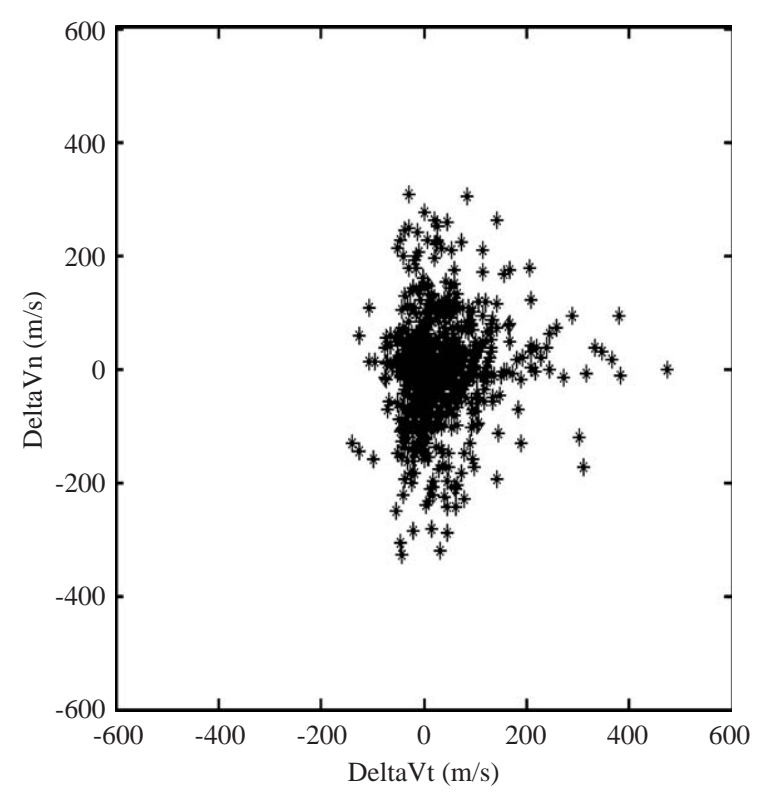

Fig. 10. $\Delta V t$ vs. $\Delta V n$ of the simulated breakup fragments of Step II rocket body.

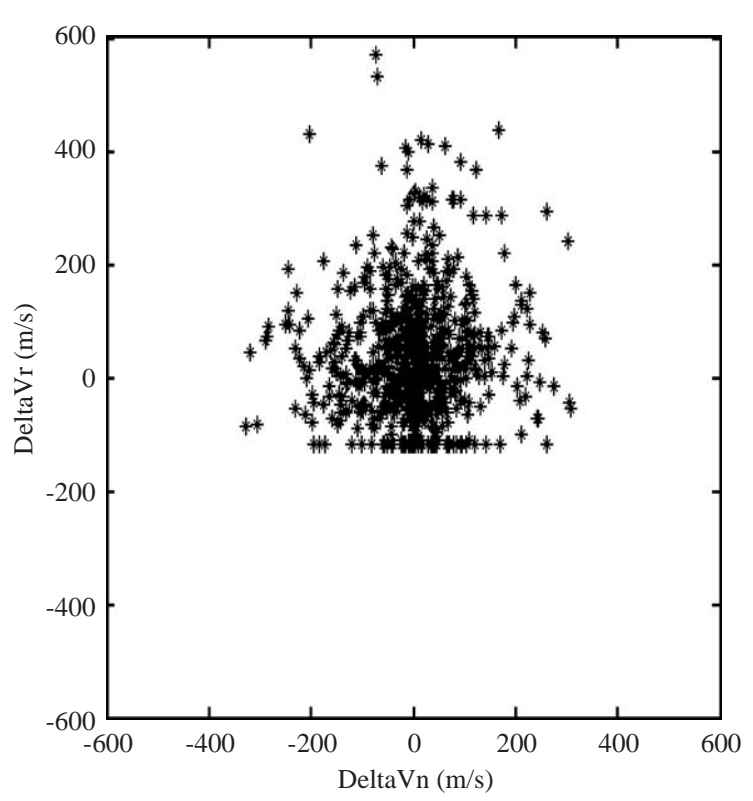

Fig. 11. $\Delta V n$ vs. $\Delta V r$ of the simulated breakup fragments of Step II rocket body.

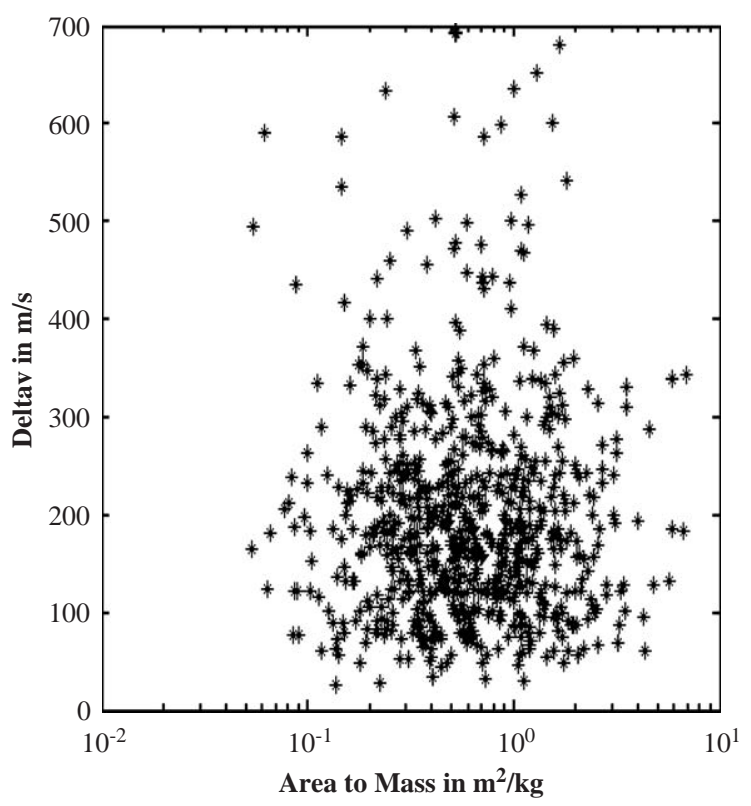

Fig. $12 . A / m$ vs. $\Delta V$ of the simulated breakup fragments of Step II rocket body. 


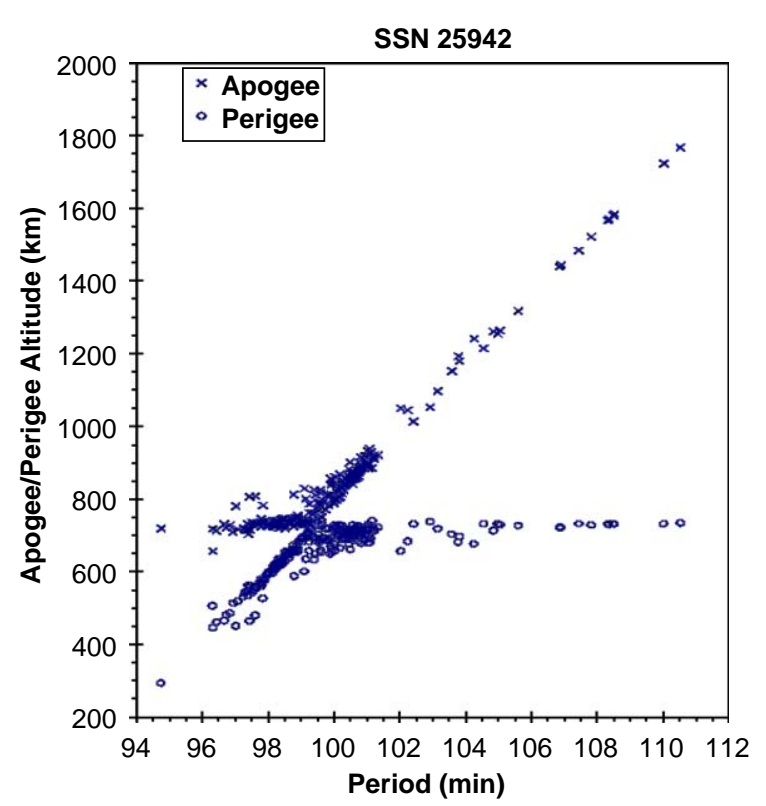

Fig. 13. CBERS-1 /SACI-1 debris clouds of 280 fragments within a day of the event as reconstructed from US SSN database $[1,18]$.

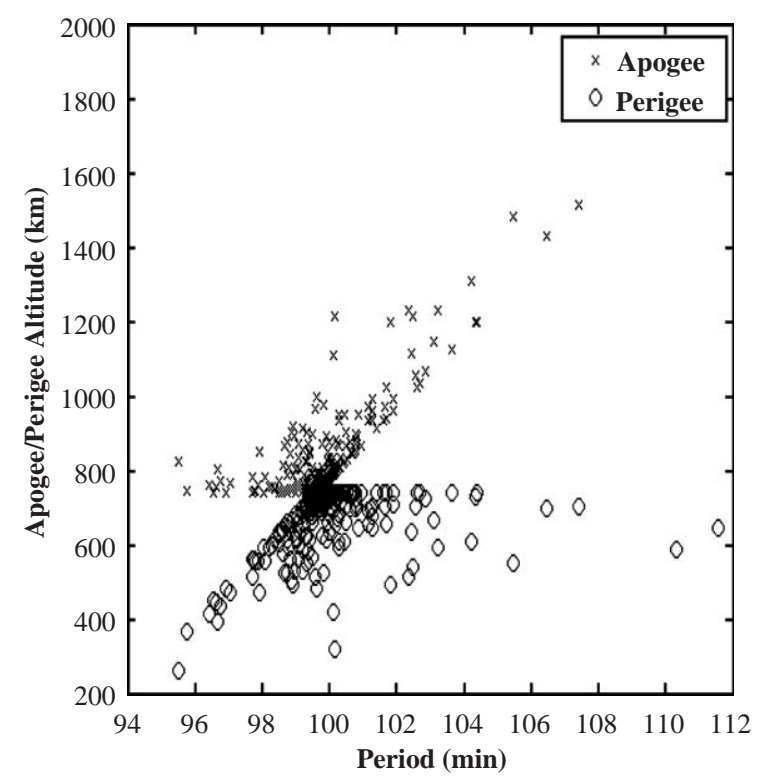

Fig. 14. Simulated debris clouds of CBERS-1 /SACI-1 at the time of breakup.

for the physical characteristics and relates them through the magnitude of $\Delta V$. It is applied to simulate some typical historical on-orbit fragmentation events. All the simulated results are comparable quite

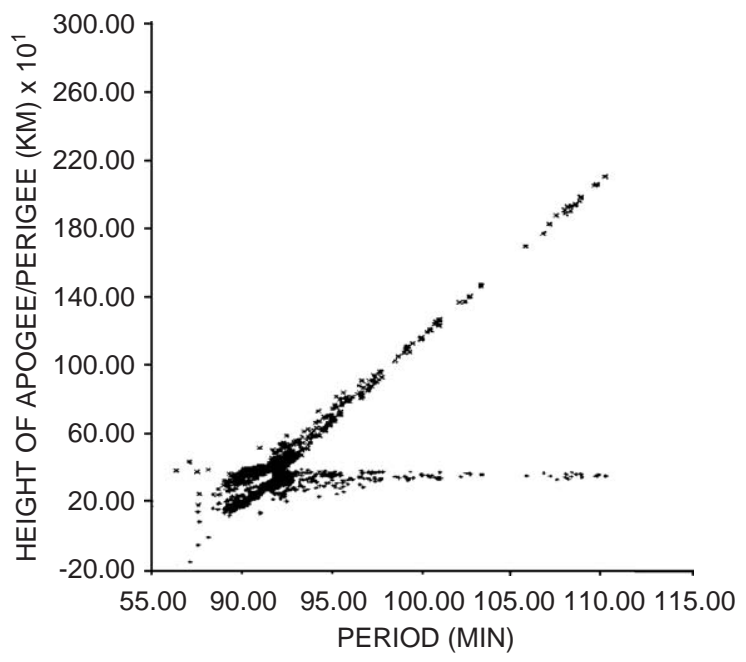

Fig. 15. Cosmos 1813 debris clouds of 846 fragments as reconstructed from PARCS radar observation taken about $10 \mathrm{~h}$ after the breakup $[1,19,20]$.

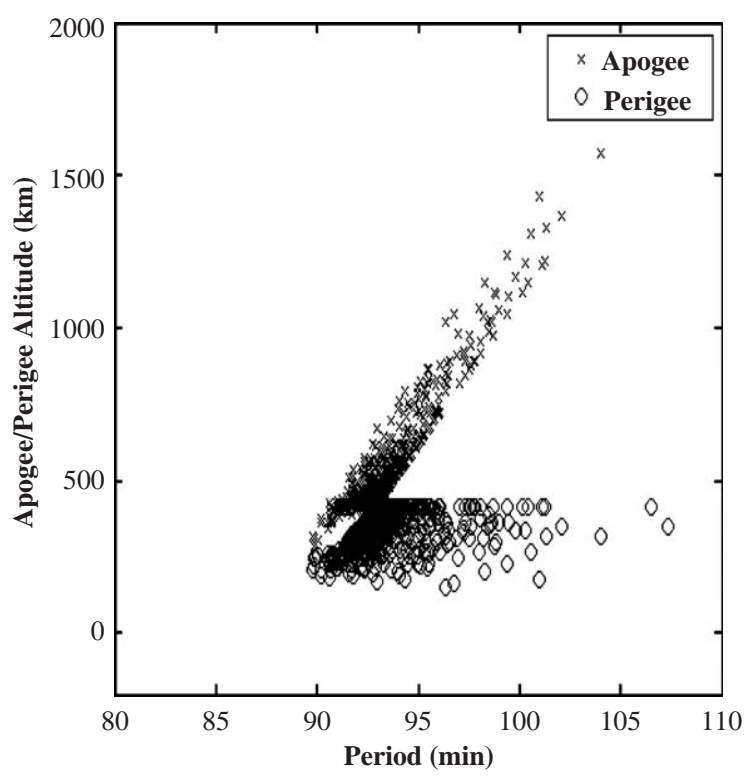

Fig. 16. Simulated debris clouds of Cosmos 1813 after $10 \mathrm{~h}$ of breakup.

well with observations both at the time of breakup and at a later epoch. It is thus demonstrated that ASSEMBLE simulates the ensemble of fragment characteristics quite well and the incremental velocity 


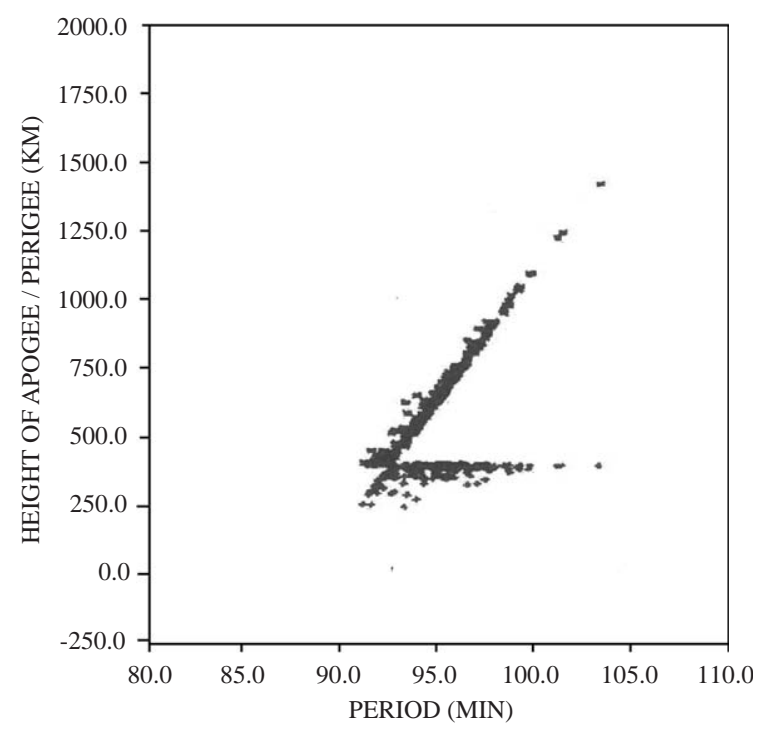

Fig. 17. Debris clouds of Cosmos 1813 after $10 \mathrm{~h}$ of breakup. Extracted from Ref. [6].

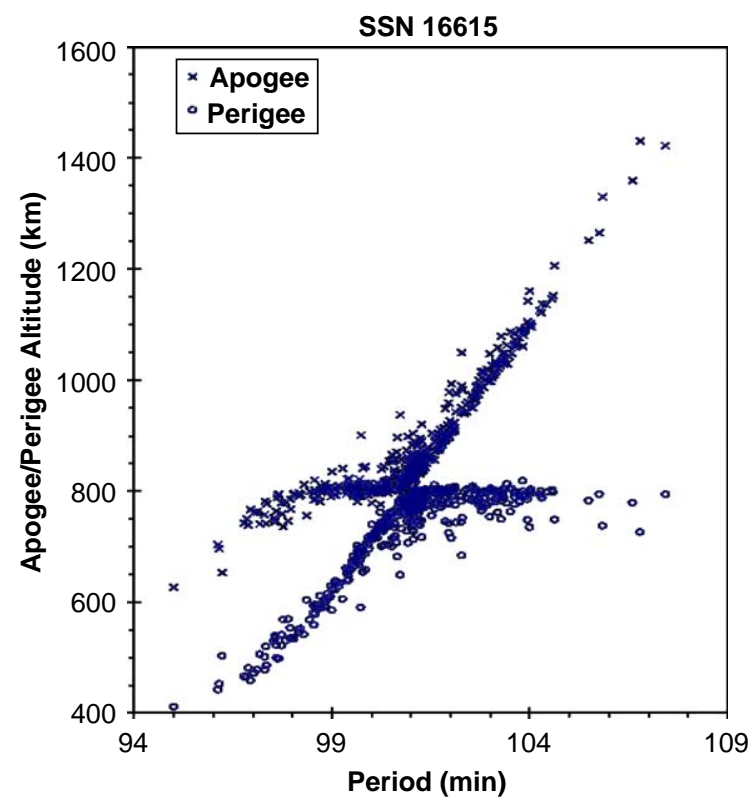

Fig. 18. SPOT I rocket body debris clouds of 463 fragments, 3 months after the event as reconstructed from US SSN database $[1,21]$.

distribution of the fragments is a consequence rather than imposed through empirical relations and the clouds remain around and much closer to the parent body than in earlier models.

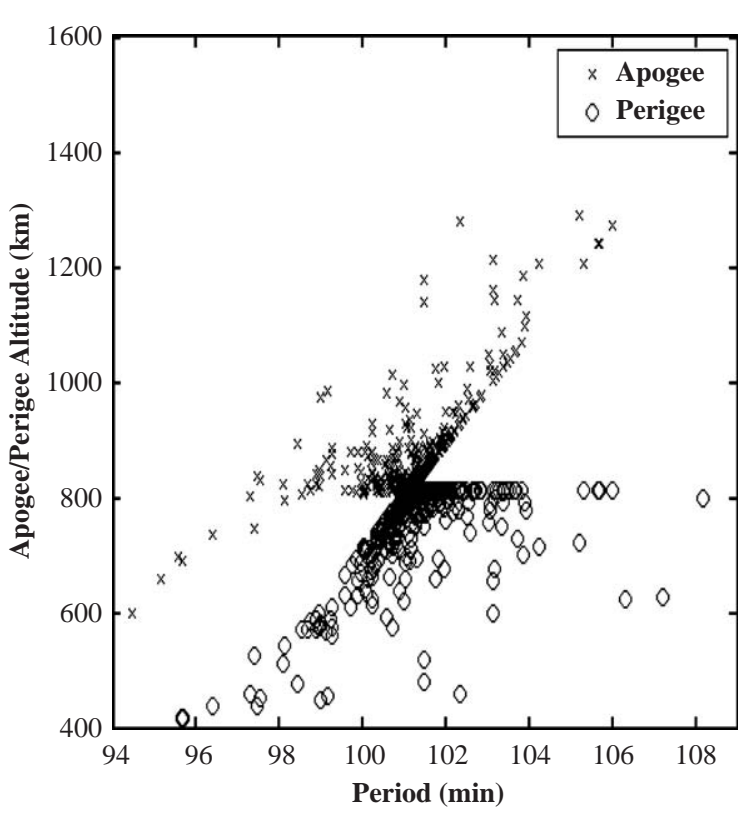

Fig. 19. SPOT I rocket body debris clouds of 463 fragments, 3 months after the event as simulated by ASSEMBLE model.

\section{Acknowledgements}

One of the authors (Anilkumar) would like to acknowledge the support provided by Vikram Sarabhai Space Centre, Indian Space Research Organization, in carrying out this research.

\section{Appendix A. Nomenclature}

$\begin{array}{ll}a & \text { semi-major axis } \\ A & \text { area of cross section } \\ A / m & \text { area to mass ratio } \\ B & \text { ballistic coefficient } \\ B^{*} & \text { term in TLE set related to ballis- } \\ & \text { tic coefficient } \\ C_{\mathrm{d}} & \text { drag coefficient } \\ d & \text { size of the fragment (m) } \\ e & \text { eccentricity } \\ f & \text { functional form of probability } \\ & \text { density function (PDF) } \\ F & \text { cumulative distribution function } \\ i & \text { inclination } \\ m & \text { mass of the fragment } \\ M & \text { mass of the parent object }\end{array}$




\begin{tabular}{|c|c|}
\hline$N$ & $\begin{array}{l}\text { number of debris fragments also } \\
\text { number of measurements }\end{array}$ \\
\hline$N_{m}$ & $\begin{array}{l}\text { cumulative number of fragments } \\
\text { with mass } m \text { or greater }\end{array}$ \\
\hline$f_{m}$ & $\begin{array}{l}\text { the ratio of the reference mass of } \\
1000 \mathrm{~kg} \text { to the parent mass } M\end{array}$ \\
\hline$\Delta V$ & $\begin{array}{l}\text { incremental velocity imparted to } \\
\text { the fragment with respect to the } \\
\text { parent which is equivalent to }\end{array}$ \\
\hline$\Delta V_{t}, \Delta V_{n}, \Delta V_{r}$ & $\begin{array}{l}\sqrt{\Delta v_{t}^{2}+\Delta v_{n}^{2}+\Delta v_{r}^{2}}(\mathrm{~m} / \mathrm{s}) \\
\text { incremental velocity in the trans- } \\
\text { verse, normal and radial direc- } \\
\text { tions, respectively, with respect } \\
\text { to the orbital plane }(\mathrm{m} / \mathrm{s})\end{array}$ \\
\hline$\Delta P$ & $\begin{array}{l}\text { deviation of the orbital period of } \\
\text { the fragments from the period of } \\
\text { the parent body ( } \mathrm{min} \text { ) }\end{array}$ \\
\hline$\Delta i$ & $\begin{array}{l}\text { deviation of the inclination of the } \\
\text { fragments from the inclination of } \\
\text { the parent body (deg) }\end{array}$ \\
\hline$\theta$ & true anomaly (deg) \\
\hline$\chi$ & azimuth (deg) \\
\hline$\varphi$ & elevation (deg) \\
\hline$\mu$ & $\begin{array}{l}\text { location parameter of the distri- } \\
\text { bution }\end{array}$ \\
\hline$\lambda$ & $\begin{array}{l}\text { scale parameter of the distribu- } \\
\text { tion }\end{array}$ \\
\hline$\rho$ & atmospheric density \\
\hline$b_{0}$ & density at a reference altitude \\
\hline
\end{tabular}

\section{Appendix B. Relationship of number to mass for the fragments}

An example of the relation that can be utilized in the present model is the rescaled relations of $\mathrm{Su}$ and Kessler for low intensity explosions $[14,22]$ :

$N= \begin{cases}171 \exp \left(-0.02056 \sqrt{m f_{m}}\right) & \text { for } m>1936 \mathrm{~g} / f_{m}, \\ 869 \exp \left(-0.05756 \sqrt{m f_{m}}\right) & \text { for } m<1936 \mathrm{~g} / f_{m},\end{cases}$

where $m$ is the mass of the fragments in grams, $M$ the mass of the breakup object in grams, $N$ the cumulative number of fragments with mass $m$ or greater, and $f_{m}$ the ratio of the reference mass of $1000 \mathrm{~kg}$ to the parent mass $M$.

\section{B.1. Association of $\Delta V$ to mass of the fragments}

In this model the delta velocities are associated with the size of the debris (and hence to the mass of the debris directly) utilizing the Reynolds's relation [22]:

$\log (\Delta V)=-0.0676(\log d)^{2}-0.804(\log d)-1.514$,

where $d$ is the size of the fragment in meters and $\Delta V$, the incremental velocity in $\mathrm{km} / \mathrm{s}$, which describes the peak in the velocity distribution curve. In order to provide dispersion in $\Delta V$, the velocity is obtained randomly from a triangular distribution whose minimum is 0.1 times and maximum is 1.3 times the peak $\Delta V$.

\section{Appendix C. Selection of scale parameters}

\section{C.1. Laplace distribution}

Laplace distribution is a bilateral exponential distribution, that is, the signed analogue of exponential distribution. This is a distribution useful for random variables from a distribution that is more peaked than a Normal distribution and is characterized by two parameters: location parameter- $\mu$, and scale parameter- $\lambda$. The probability density function $f(x)$ and the cumulative distribution function $F(x)$ are, respectively,

$f(x)=\frac{1}{2 \lambda} \exp \left(-\frac{|x-\mu|}{\lambda}\right)$,

$F(x, \mu, \lambda)= \begin{cases}\frac{1}{2} \exp \left(\frac{x-\mu}{\lambda}\right), & x \leqslant \mu, \\ \frac{1}{2} \exp \left(\frac{\mu-x}{\lambda}\right), & x>\mu,\end{cases}$

with mean $=\mu$ and variance $=2 \lambda^{2}$. Assuming $F=0.5+$ $\alpha$ and $(\mu-x)=\delta, \lambda$ can be obtained as $\lambda=\delta / \log (2 \alpha)$.

\section{C.2. Lognormal distribution}

The Lognormal distribution is the distribution of a random variable, which in log space is following a Normal distribution. The Lognormal distribution is always right skewed. This is a two-parameter distribution, with mean and standard deviations of the variable in natural log space. The two parameters are location parameter- $\mu$-and scale parameter- $\lambda$. 
The probability density function $f(x)$ and the cumulative distribution function $F(x)$ are, respectively,

$$
\begin{aligned}
& f(x)=\frac{1}{\lambda x \sqrt{2 \pi}} \exp \left(-\left(\frac{\log (x)-\mu}{\lambda}\right)^{2}\right), \\
& F(t) \int_{0}^{t} \frac{1}{\lambda x \sqrt{2 \pi}} \exp \left(-\left(\frac{\log (x)-\mu}{\lambda}\right)^{2}\right) \mathrm{d} x,
\end{aligned}
$$

with mean $=\exp \left(\mu+\lambda^{2} / 2\right)$ and variance $=\exp (2 \mu+$ $\left.\lambda^{2}\right)\left(\exp \left(\lambda^{2}\right)-1\right)$. Evaluating $F$ for known location and range $(0, t)$ the value of $\lambda$ can be determined for a probabilistic confidence level of $\alpha \%$.

\section{References}

[1] P.D. Anz-Meador, History of On-Orbit Satellite Fragmentations, JSC 29517, NASA, July 2001.

[2] Orbital Debris Quarterly News, vol. 7, Issue 1, January 2002.

[3] W. Feller, An Introduction to Probability theory and its Applications, vol. 1, third ed., Wiley Eastern Limited, NewDelhi, 1950.

[4] N.L. Johnson, D.S. McKnight, Artificial Space Debris, Orbit Book Company, 1987.

[5] A.S. Ganeshan, M.R. Ananthasayanam, Simulation and modeling of orbital debris environment by equivalent breakups, Advances in Space Research 19 (2) (1997).

[6] R. Jehn, Modeling Debris Clouds, Shaker Verlag, 1996.

[7] Upgrade of ESA's MASTER Model, Final Report, MASGEN-FR, Revision 1.0, June 2000.

[8] R.C. Reynolds, Documentation of Program EVOLVE: A Numerical Model to Compute Projections of the Man-Made Orbital Debris Environment, OD91-002-U-CSP, 1991.

[9] N.L. Johnson, NASA's New Breakup Model of EVOLVE 4.0, XXXIII COSPAR Scientific Assembly, Warsaw, Poland, July 2000.
[10] Upgrade of ESA's Master Model, Final Report, MAS-GENFR, Revision 1.3.1, December 2002.

[11] A.K. Anilkumar, M.R. Ananthasayanam, P.V. Subba Rao, Analysis and Modeling of PSLV-TES Mission Spent Upper Stage Breakup in LEO, Report 2002 FM4, Department of Aerospace Engineering, Indian Institute of Science, Bangalore, February 2002.

[12] M.P. McLaughlin, A Tutorial on Mathematical Modeling, 1999. http://www.geocities.com/ mikemclaughlin/software/ Regress_plus.html.

[13] V.K. Rohatgi, An Introduction to Probability Theory and Mathematical Statistics, Wiley Eastern Limited, New Delhi, 1990.

[14] S.Y. Su, D.J. Kessler, Contributions of explosions and future collision fragments to the orbital debris environment, Advances in Space Research 5 (2) (1985) 25-34.

[15] N.L. Johnson, Major Satellite Breakup in June, Orbital Debris Quarterly News, NASA JSC, September 1996, pp. 2 and 11.

[16] D. King-Hele, Satellite Orbits in an Atmosphere: Theory and Applications, Blackie, Glasgow and London, 1987.

[17] P. Anz-Meador, HAPS Debris Separation Velocity Distribution, The Orbital Debris Quarterly News 5 (2) April 2000.

[18] W.X. Zang, S.Y. Liao, Analyzing the Cause of LM-4 (A)'s Upper Stage's Disintegration and the Countermeasures, 5th International Conference of Pacific Basin Societies, 6-9 June 1993, Shanghai.

[19] R.L. Kling, J.S. Dowdy, The Fragmentation of Kosmos 1813, Technical Report CS87-LKD-004, Teledyne Brown Engineering, Colorado Springs, May 1987.

[20] N.L. Johnson, et al., History of Soviet/Russian Satellite Fragmentations-A Joint U.S.-Russian Investigation, Kaman Sciences Corporation, October 1995.

[21] N.L. Johnson, A Preliminary Analysis of the Fragmentation of the SPOT I Ariane Third Stage, Technical Report CS87LKD-003, Teledyne Brown Engineering, Colorado Springs, March 1987.

[22] R.C. Reynolds, A Review of Orbital Debris Environment Modeling at NASA/JSC, Orbital Debris Conference, AIAA-90-1355, Baltimore, April 1990. 